\title{
Efeitos da COVID-19 entre gestantes pretas e pardas: Revisão integrativa da
}

\section{literatura}

\author{
Effects of COVID-19 among black and mulatto pregnant women: Integrative literature review \\ Efectos del COVID-19 entre las embarazadas negras y mulatas: Revisión de literatura integradora
}

Recebido: 27/04/2021 | Revisado: 05/05/2021 | Aceito: 07/05/2021 | Publicado: 18/05/2021

\author{
Gustavo Gonçalves dos Santos \\ ORCID: https://orcid.org/0000-0003-1615-7646 \\ Universidade Estadual Paulista "Júlio de Mesquita Filho", Brasil \\ E-mail: gustavo.goncalves-santos@unesp.br \\ Luis Henrique de Andrade \\ ORCID: https://orcid.org/0000-0003-2982-5252 \\ Universidade Estadual Paulista "Júlio de Mesquita Filho", Brasil \\ E-mail: 1h.andrade@unesp.br \\ Anna Paula Frassom da Silva Magaton \\ ORCID: https://orcid.org/0000-0002-6980-3587 \\ Universidade Federal de São Paulo, Brasil \\ E-mail: anaenfermeira30@gmail.com \\ Cristina Maria Garcia de Lima Parada \\ ORCID: https://orcid.org/0000-0002-9597-3635 \\ Universidade Estadual Paulista “Júlio de Mesquita Filho”, Brasil \\ E-mail: cristina.parada@unesp.br
}

\begin{abstract}
Resumo
Objetivo: identificar e analisar a produção científica sobre a evolução da COVID-19 em gestantes/puérperas segundo cor da pele/raça. Metodologia: trata-se de Revisão Integrativa da Literatura (RIL), com busca nas bases de dados EMBASE, LILACS, portal PUBMED/MEDLINE e Web of Science realizada em fevereiro de 2021. A partir da estratégia PICO foi construída a pergunta de pesquisa: Há evidências que gestantes/puérperas pretas com COVID-19, tratadas em ambiente hospitalar, quando comparadas às gestantes brancas, evoluem com mais gravidade (necessidade de internação em Unidade de Terapia Intensiva/Óbito)? Foram utilizados os termos indexados no vocabulário estruturado dos Descritores em Ciências da Saúde (DeCS) e Medical Subject Headings (MeSH terms): "COVID-19" OR "2019-nCoV" AND "Gestante" OR "Cuidado pré-natal" OR "gravidez" AND "Raça" OR "Grupos Raciais" OR "Racismo" OR "Iniquidades Sociais" OR "Afro-americano" AND "Hospitalização" OR "Unidade de Internação" AND "Morte" OR "Causas de Morte" OR "Morte Materna" OR "Unidades de Terapia Intensiva". Resultados: foram identificados seis estudos, realizados nos Estados Unidos da América (três estudos), Reino Unido (um estudo) e Brasil (dois estudos) e apenas um deles foi classificado com nível de evidência 4, sendo os demais de nível 6. Todos os estudos eram observacionais, muitas vezes com amostras pequenas e grupos étnicos variados, não permitindo conclusões efetivas. Dois estudos brasileiros que utilizaram banco de dados secundários e de base populacional encontraram a raça/cor preta como fator de risco para COVID-19 grave. Conclusão: com os estudos analisados, não foi possível estabelecer clara relação entre raça/cor e desfechos negativos (necessidade de internação em Unidade de Terapia Intensiva/óbito) da COVID-19 entre gestantes/puérperas.
\end{abstract}

Palavras-chave: COVID-19; Gestante; Raça; Racismo; Morte.

\begin{abstract}
Objective: to identify and analyze the scientific production on the evolution of COVID-19 in pregnant/puerperal women according to skin color/race. Methodology: this is an Integrative Literature Review (ILR), with a search in the EMBASE, LILACS, PUBMED/MEDLINE and Web of Science databases conducted in February 2021. From the PICO strategy the research question was constructed: Is there evidence that black pregnant women/puerperal women with COVID-19, treated in a hospital setting, when compared to white pregnant women, evolve with more severity (need for admission to the Intensive Care Unit/death)? The terms indexed in the structured vocabulary of the Health Sciences Descriptors (DeCS) and Medical Subject Headings (MeSH terms) were used: "COVID-19" OR "2019nCoV" AND "Pregnant Woman" OR "Prenatal Care" OR "pregnancy" AND "Race" OR "Racial Groups" OR "Racism" OR "Social Inequities" OR "African American" AND "Hospitalization" OR "Inpatient Unit" AND "Death" OR "Causes of Death" OR "Maternal Death" OR "Intensive Care Units". Results: Six studies were identified, conducted in the United States of America (three studies), United Kingdom (one study), and Brazil (two studies), and only one of them was classified as level of evidence 4, the others as level 6 . All studies were observational, often with small samples and varied ethnic groups, not allowing effective conclusions. Two Brazilian studies that used
\end{abstract}


secondary, population-based databases found black race/color to be a risk factor for severe COVID-19. Conclusion: With the studies analyzed, it was not possible to establish a clear relationship between race/color and negative outcomes (need for admission to the intensive care unit/death) of COVID-19 among pregnant/puerperal women.

Keywords: COVID-19; Pregnant Woman; Race; Racism; Death.

\section{Resumen}

Objetivo: identificar y analizar la producción científica sobre la evolución de la COVID-19 en gestantes/puérperas del cuerpo de la pelea/raza. Metodología: se trata de una Revisión Integradora de Literatura (RIL), con una búsqueda en las bases de datos EMBASE, LILACS, PUBMED/MEDLINE y Web of Science realizada en febrero de 2021. Sobre la base de la estrategia PICO, se construyó la pregunta de investigación: ¿Existen pruebas de que las mujeres negras embarazadas/puerperas con COVID-19, tratadas en un entorno hospitalario, en comparación con las mujeres blancas embarazadas, evolucionan con mayor gravedad (necesidad de ingreso en la Unidad de Cuidados Intensivos/muerte)? Se utilizaron los términos indexados en el vocabulario estructurado de los Descriptores en Ciencias de la Salud (DeCS) y los Encabezamientos de Materia Médica (términos MeSH): "COVID-19" OR "2019-nCoV" AND "Pregnant Woman" OR "Prenatal Care" OR "pregnancy" AND "Race" OR "Racial Groups" OR "Racism" OR "Social Inequities" OR "African American" AND "Hospitalization" OR "Inpatient Unit" AND "Death" OR "Causes of Death" OR "Maternal Death" OR "Intensive Care Units". Resultados: Se identificaron seis estudios, realizados en Estados Unidos de América (tres estudios), Reino Unido (un estudio) y Brasil (dos estudios) y sólo uno de ellos fue clasificado como nivel de evidencia 4, los demás como nivel 6. Todos los estudios fueron observacionales, a menudo con muestras pequeñas y grupos étnicos variados, lo que no permite obtener conclusiones eficaces. Dos estudios brasileños que utilizaron bases de datos secundarias basadas en la población encontraron que la raza/color negro es un factor de riesgo de COVID-19 grave. Conclusión: con los estudios analizados, no fue posible establecer una relación clara entre raza/color y resultados negativos (necesidad de ingreso en la unidad de cuidados intensivos/muerte) de COVID-19 entre las mujeres embarazadas/puerperas.

Palabras clave: COVID-19; Gestante; Raza; Racismo; Muerte.

\section{Introdução}

Em dezembro de 2019 se descreve um novo coronavírus, após casos de a doença ser registrados originalmente na China. Infecção altamente contagiosa e fatal, no período de três meses se disseminou de maneira rápida mundialmente (Brasil, 2020a).

Em 11 de março de 2020 com a progressão dos casos de forma avassaladora, a Organização Mundial da Saúde (OMS) declarou pandemia de COVID-19 (World Health Organization, 2020 - SR51). Um mês depois, globalmente, já estavam confirmados 1.521.252 casos e 92.798 óbitos (World Health Organization, 2020 - SR81). No Brasil, na mesma data, havia 19.638 casos confirmados e 1.056 óbitos, com taxa de letalidade de 5,4\% (Brasil, 2020b) e cinco meses depois, em agosto de 2020, dados globais indicavam que o país já ocupava a segunda posição mundial em relação ao número de casos e de óbitos, respectivamente, 3.317.096 e 107.232 (Brasil, 2020b).

Diante da evolução da pandemia de COVID-19, tornou-se importante a definição de grupos com maior risco de evolução grave da doença e, nestes, têm sido incluídas as gestantes e puérperas (Brasil, 2020c). A gestação é um período com diversas alterações fisiológicas, e as mulheres gestantes e puérperas, durante as infecções causadas por outros coronavírus, como a Severe Acute Respiratory Syndrome Coronavirus (SARSCoV) e Middle East Respiratory Syndrome Coronavírus (MERSCoV), ocorridas em 2003 e 2012, respectivamente, apresentaram complicações variadas, como: febre, tosse, dispneia e necessidade de Unidade de Terapia Intensiva (UTI) (Wong et al., 2003; Alfaraj; AL-Tawfiq; Memish, 2019), justificando a inclusão no grupo de risco para o SARS-CoV-2. Na maioria das vezes os sintomas apresentados são leves, com febre e tosse seca, porém, em mulheres na segunda metade da gestação, outros sintomas podem aparecer com intensidade variada, como fadiga, dispneia, diarreia, congestão nasal e coriza, sendo também possível evolução para complicações graves, como a Síndrome Respiratória Aguda Grave (Zaigham; Andersson, 2020).

A literatura científica aponta que gestantes com infecção por SARS-CoV-2, que evoluem para quadro grave associado à comorbidade, apresentam probabilidade aumentada de evoluírem para parto por operação cesariana emergencial ou para 
trabalho de parto prematuro, o que eleva o risco de morbimortalidade materna e neonatal (Li et al.; 2020). Outros cinco estudos foram realizados com gestantes chinesas no início da Pandemia: o primeiro, realizado com nove gestantes, descreveu quadro clínico semelhante à de mulheres não grávidas, sendo que quatro gestantes $(44,4 \%)$ evoluíram para parto prematuro (Chen et al., 2020); em outros dois estudos, com sete gestantes infectadas no final da gravidez (Yu et al., 2020) e 15 gestantes infectadas nos três trimestres gestacionais (Liu, 2020a), os autores encontraram bons resultados maternos e neonatais; em caso único relatado, gestante com 30-31 semanas de gestação evoluiu para parto por operação cesariana, nascendo um bebê saudável, sem evidência de infecção (Wang et al., 2020) e, por fim, um estudo realizado com 13 gestantes concluiu que estas são suscetíveis à infecção e que na vigência da doença pode haver aumento no risco a saúde do binômio (Liu et al., 2020b).

Na América Central foi reportada em Carta ao Editor, a descrição de quadro leve de COVID-19 em uma gestante, com evolução para trabalho de parto prematuro espontâneo, em idade gestacional de 32 semanas (Zambrano et al., 2020). Nos Estados Unidos da América (EUA), de sete casos confirmados em um centro de atendimento terciário, dois $(28,6 \%)$ necessitaram de internação na UTI, sendo que ambas gestantes eram assintomáticas na internação (Breslin et al., 2020).

Estudo americano do Centers for Diseases Control and Prevention (CDC) (Ellington; Strid; Tong et al., 2020), estudo sueco (Collin; Bystrom; Carnahan; Ahrne, 2020) e outros realizados na Bélgica e França (Vivanti; Mattern; Vauloup-Fellous; Jani; Rigonnot; El Hachem et al., 2020) apontam risco aumentado de complicações e internação em UTI, além de maior necessidade de ventilação mecânica em gestantes, mas não apontaram maior risco de morte materna. As mortes maternas parecem, de fato, mais frequentes nos países de baixa e média renda e seriam decorrentes de falhas graves do sistema de saúde, aliadas aos determinantes sociais do processo saúde-doença. Isso também tem sido visto em outros países da América Latina (Amorim; Takemoto; Fonseca, 2020), sobretudo no México, país que mantém um sistema de notificação de casos eficiente (Lumbreras-Marquez; Campos-Zamora; Lizaola-Diaz; Farber, 2020).

No contexto da América Latina a situação brasileira é preocupante. Levantamento realizado em meados de 2020 sobre os óbitos maternos por COVID-19 no mundo encontrou 160 casos registrados na literatura internacional e indicou que de cada 10 óbitos oito aconteciam no Brasil (Nakamura-Pereira et al., 2020). Outro estudo desse mesmo grupo de pesquisadores, realizado à mesma época e divulgado no formato de Comunicação Breve em julho de 2020, apresentou que 124 mulheres grávidas ou puérperas haviam morrido no país devido a COVID-19, resultando em taxa de mortalidade de 12,7\%. Assim, o número de casos superava o total de mortes maternas relacionadas à COVID-19 do restante do mundo, destacando que 22,6\% das mulheres com esse desfecho não haviam sido admitidas em UTI, 64\% haviam recebido ventilação invasiva e 14,6\% não haviam tido acesso à intubação. $\mathrm{O}$ mesmo estudo informou terem identificado nas gestantes/puérperas diabetes, doenças cardiovasculares e obesidade como condições significativamente associadas à mortalidade, semelhante ao que ocorre na população em geral. (Takemoto; Menezes; Andreucci; Nakamura-Pereira; Amorim; Katz; Knobel, 2020).

Considerando as lacunas do conhecimento frente à ocorrência da COVID-19, o presente estudo tem por foco um grupo específico de mulheres, aquelas que se autodeclaram pretas ou pardas. Assim, tem-se por objetivo identificar e analisar a produção científica na literatura nacional e internacional sobre a evolução da COVID/19 em gestantes/puérperas segundo cor da pele/raça. O interesse por esse tema decorre da possível identificação de maior risco entre essas mulheres, que em geral vivenciam condição de desigualdade social e dificuldade de acesso aos serviços de saúde. Em dados divulgados pela Organização das Nações Unidas (ONU), observou-se que a mortalidade materna em mulheres pretas devido à COVID-19 foi quase duas vezes maior que a observada em mulheres brancas (ONU Mulheres; ONU, 2020). Pesquisadores têm apontado que os desdobramentos da pandemia da COVID-19 em uma sociedade estruturada pelo racismo penalizam grupos vulneráveis (Goes, 2020), como as mulheres pretas, em decorrência de sua inserção social, política, econômica e cultural (Santos, 2020). 


\section{Metodologia}

Trata-se de Revisão Integrativa da Literatura (RIL), método que tem como objetivo reunir conhecimento sobre determinado assunto, permitindo análise e síntese dos resultados de estudos independentes (Ganong, 1987; Souza; Silva \& Carvalho, 2010), denominada integrativa porque fornece informações amplas sobre o tema, construindo, assim, um corpo de conhecimento (Ercole; Melo; Alcoforado, 2014). O percurso metodológico para a realização da RIL seguiu seis etapas: 1Seleção da hipótese; 2- definição da amostra, a partir do estabelecimento de critérios para inclusão e exclusão dos artigos; 3 coleta de dados, com descrição das características dos estudos; 4- análise minuciosa dos estudos, realizada a partir da definição do seu nível de evidência; 5- interpretação dos resultados e 6- apresentação da RIL (Galvão; Sawada; \& Trevizan, 2004).

Para definição da pergunta do estudo, utilizou-se a estratégia PICO, acrônimo para Paciente, Intervenção, Comparação e Outcomes (desfecho), em que: "P" - gestantes/puérperas negras com COVID-19; "I" - tratamento hospitalar; "C" - comparação entre gestantes brancas e pretas/pardas; e "O" - desfechos: gravidade da evolução da COVID-19, resultando na seguinte pergunta: Há evidências que gestantes/puérperas negras com COVID-19, tratadas em ambiente hospitalar, quando comparadas às gestantes brancas, evoluem com mais gravidade (necessidade de UTI/Óbito)?

Definiram-se como critérios de inclusão dos estudos: artigos que respondessem à questão do estudo; disponíveis na íntegra em meio eletrônico gratuitamente; em idioma inglês ou português e publicados a partir do ano de 2020. Foram critérios de exclusão: artigos em duplicidade e com desenho de RIL.

A busca na literatura se deu no mês de fevereiro de 2021, nas seguintes bases de dados: Excerpta Medica dataBASE (EMBASE) da Elsevier Scientific Publications, Web of Science (WoS), Literatura Latino-americana e do Caribe em Ciências da Saúde (LILACS) e portal National Library of Medicine (PUBMED)/Medical Literature Analysis and Retrievel System Online (MEDLINE). Para a coleta de dados foram utilizados termos indexados no vocabulário estruturado dos Descritores em Ciências da Saúde (DeCS) e Medical Subject Headings (MeSH terms) utilizando os termos: "COVID-19” OR "2019-nCoV” AND “Gestante” OR “Cuidado pré-natal” OR “Gravidez” AND “Raça” OR “Grupos Raciais” OR "Racismo” OR "Iniquidades Sociais" OR “Afro-americano” AND “Hospitalização” OR “Unidade de Internação” AND “Morte” OR "Causas de Morte” OR "Morte Materna” OR “Unidades de Terapia Intensiva”.

Para seleção dos artigos, iniciou-se a busca na base EMBASE, resultando em 21 artigos, sendo que após leitura do título e resumo, foram selecionados seis para leitura completa e, após leitura crítica e minuciosa, apenas um artigo foi incluído na revisão. Em LILACS, a busca resultou em 13 artigos, sendo que, após leitura do título e resumo, dois foram selecionados para leitura crítica e minuciosa, nenhum deles incluído na revisão, por não responderem à questão da pesquisa. Em PUBMED/MEDLINE, a busca resultou em 17 artigos e após leitura do título e resumo, 10 foram selecionados para leitura crítica e minuciosa, sendo cinco incluídos na revisão. Por fim, em Web of Science, 21 artigos foram inicialmente selecionados para leitura do título e resumo e, após a leitura crítica e minuciosa, nenhum foi incluído na presente revisão, pois também não respondiam à questão da pesquisa (Figura 1).

Em síntese, compuseram a RIL seis artigos, sendo que as publicações analisadas foram originadas de estudos observacionais. Para análise dos níveis de evidência dos artigos, considerou-se: 1- revisões sistemáticas ou metanálises; 2estudos controlados randomizados; 3- estudos controlados não randomizados; 4- estudos de coorte e casos-controle; 5- revisões sistemáticas de estudos qualitativos ou descritivos; 6- estudos qualitativos ou descritivos e 7- opinião de experts (Melnyk e Fineout-Overholt, 2005). 
Research, Society and Development, v. 10, n. 6, e6710615531, 2021

(CC BY 4.0) | ISSN 2525-3409 | DOI: http://dx.doi.org/10.33448/rsd-v10i6.15531

Figura 1 - Fluxograma do processo de inclusão de artigos.

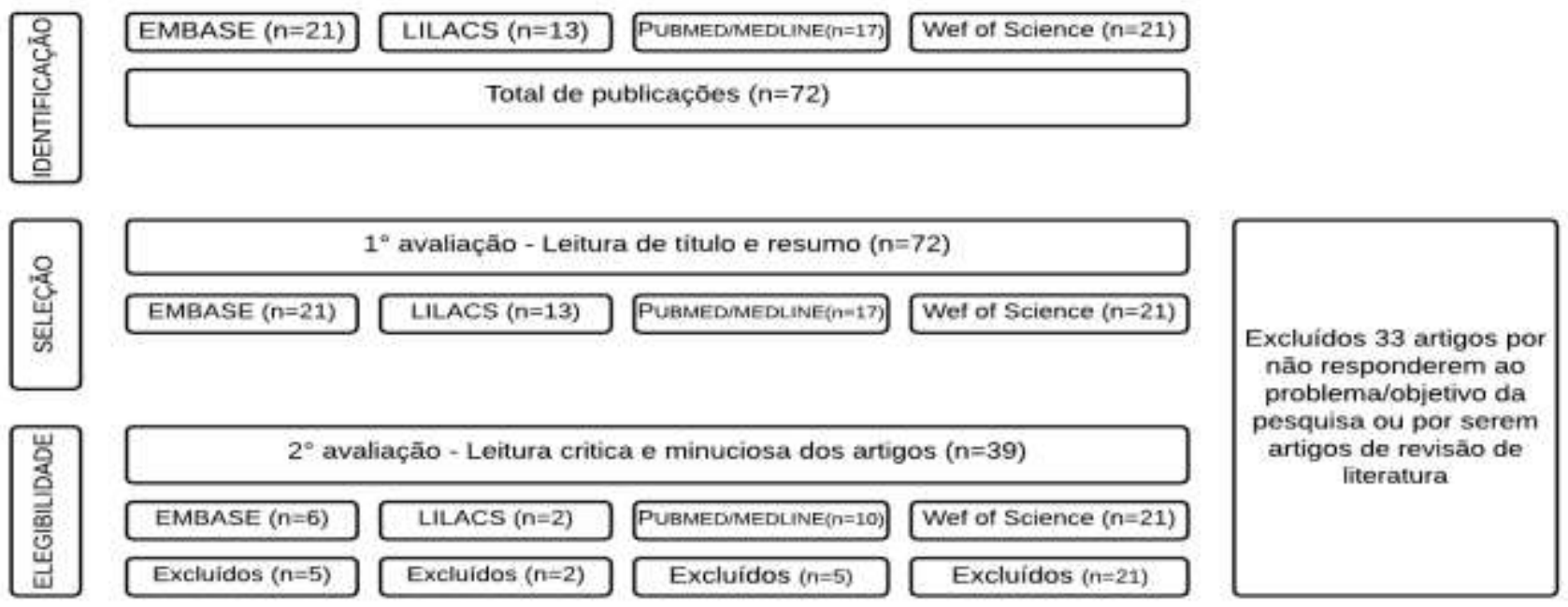

$(n=6)$ por responderem ao problema/objetivo da pesquisa, estarem disponiveis eletronicamente e por serem artigos de pesquisa originais

Fonte: Autores (2021).

Os resultados obtidos foram sistematizados em três quadros: o primeiro contém ano, autores, título, país, periódico, nível de evidência e base/portal de dados (Quadro 1); o segundo, contempla tipo de estudo, amostra e objetivos (Quadro 2); e o terceiro resultados e principais conclusões dos artigos (Quadro 3). Para facilitar a compreensão dos resultados, em todos os quadros os artigos foram identificados pela letra $\mathrm{E}$, seguida pelo número que o identificou, variando de 1 a 6 (E1... E6).

Por tratar-se de RIL, não foi necessário encaminhamento deste estudo para apreciação de Comitê de Ética em Pesquisa. 


\section{Resultados}

Quadro 1 - Caracterização dos artigos incluídos na revisão integrativa segundo ano, autores, título, país, periódico, nível de evidência e base/portal de dados.

\begin{tabular}{|c|c|c|c|c|c|c|c|}
\hline Identificação & Ano & Autores & Título & País & Periódico & $\begin{array}{l}\text { Nível de } \\
\text { evidência }\end{array}$ & Base/portal de dados \\
\hline E1 & $\begin{array}{c}202 \\
1\end{array}$ & $\begin{array}{l}\text { Lokken, E.M., Taylor, G. G., Huebner E. } \\
\text { M., Vanderloeven J., Hendrickson S., } \\
\text { Coler, B. et al. }\end{array}$ & $\begin{array}{l}\text { Higher SARS-CoV-2 infection rate in pregnant } \\
\text { patients }\end{array}$ & $\begin{array}{l}\text { Estados } \\
\text { Unidos da } \\
\text { América }\end{array}$ & $\begin{array}{c}\text { American Journal of } \\
\text { Obstetrics \& } \\
\text { Gynecology }\end{array}$ & Nível 6 & PUBMED/MEDLINE \\
\hline E2 & $\begin{array}{c}202 \\
1\end{array}$ & $\begin{array}{l}\text { Jani, S., Jacques, S.M., Qureshi, F., } \\
\text { Natarajan, G., Bajaj, S., Velumula, P., Agu, } \\
\text { C. \& Bajaj M }\end{array}$ & $\begin{array}{l}\text { Clinical characteristics of mother-infant dyad } \\
\text { and placental pathology in COVID-19 cases in } \\
\text { predominantly african american population }\end{array}$ & $\begin{array}{l}\text { Estados } \\
\text { Unidos da } \\
\text { América }\end{array}$ & AJP Reports & Nível 6 & EMBASE \\
\hline E3 & $\begin{array}{c}202 \\
0\end{array}$ & $\begin{array}{l}\text { Knight, M., Bunch, K., Vousden, N., } \\
\text { Morris, E., Simpson, N., Gale, C., et al. }\end{array}$ & $\begin{array}{l}\text { Characteristics and outcomes of pregnant } \\
\text { women admitted to hospital with confirmed } \\
\text { SARS-CoV-2 infection in UK: national } \\
\text { population based cohort study }\end{array}$ & Reino Unido & $\begin{array}{c}\text { British Medical } \\
\text { Journal }\end{array}$ & Nível 4 & PUBMED/MEDLINE \\
\hline E4 & $\begin{array}{c}202 \\
0\end{array}$ & $\begin{array}{l}\text { Santos, D.S., Menezes, M.O., Andreucci, } \\
\text { C.B., Nakamura-Pereira, M., Knobel, R., } \\
\text { Katz, L. et al. }\end{array}$ & $\begin{array}{l}\text { Disproportionate impact of COVID-19 among } \\
\text { pregnant and postpartum black women in } \\
\text { Brazil through structural racism lens }\end{array}$ & Brasil & $\begin{array}{c}\text { Clinical Infectious } \\
\text { Diseases }\end{array}$ & Nível 6 & PUBMED/MEDLINE \\
\hline E5 & $\begin{array}{c}202 \\
0\end{array}$ & $\begin{array}{l}\text { Onwuzurike, C., Diouf, K., Meadows, A.R., } \\
\text { Nour, N.M }\end{array}$ & $\begin{array}{l}\text { Racial and ethnic disparities in severity of } \\
\text { COVID-19 disease in pregnancy in the United } \\
\text { States }\end{array}$ & $\begin{array}{l}\text { Estados } \\
\text { Unidos da } \\
\text { América }\end{array}$ & $\begin{array}{l}\text { International Journal } \\
\text { of Gynecology \& } \\
\text { Obstetrics }\end{array}$ & Nível 6 & PUBMED/MEDLINE \\
\hline E6 & $\begin{array}{c}202 \\
0\end{array}$ & $\begin{array}{l}\text { Menezes, M.O., Takemoto, M.L.S., } \\
\text { Nakamura-Pereira, M., Katz, L., Amorim, } \\
\text { M.M.R., Salgado, H.O., Melo, A., Diniz, } \\
\text { C.S.G., de Sousa, L.A.R., Magalhaes, C.G., } \\
\text { Knobel, R. \& Andreucci, C.B }\end{array}$ & $\begin{array}{l}\text { Risk factors for adverse outcomes among } \\
\text { pregnant and postpartum women with acute } \\
\text { respiratory distress syndrome due to COVID- } \\
19 \text { in Brazil }\end{array}$ & Brasil & $\begin{array}{l}\text { International Journal } \\
\text { of Gynecology \& } \\
\text { Obstetrics }\end{array}$ & Nível 6 & PUBMED/MEDLINE \\
\hline
\end{tabular}

Fonte: Autores (2021). 
Research, Society and Development, v. 10, n. 6, e6710615531, 2021

(CC BY 4.0) | ISSN 2525-3409 | DOI: http://dx.doi.org/10.33448/rsd-v10i6.15531

Quadro 2 - Caracterização dos artigos incluídos na revisão integrativa segundo tipo de estudo, amostra e objetivos.

\begin{tabular}{|c|c|c|c|}
\hline Identificação & Tipo de estudo & Amostra & Objetivos \\
\hline E1 & Estudo descritivo & $\begin{array}{l}240 \text { grávidas com SARS-CoV-2 entre } 1 \text { de março e } 30 \text { de junho de } \\
2020 \text {, atendidas em } 35 \text { hospitais de Washington. }\end{array}$ & $\begin{array}{l}\text { Estimar a taxa de infecção de } S A R S-C o V-2 \text { na gravidez e examinar as disparidades por } \\
\text { raça/etnia no estado de Washington. }\end{array}$ \\
\hline E2 & Estudo descritivo & 34 díades mãe-bebê positivas para COVID-19. & Avaliar os desfechos clínicos em díades mãe-bebê na vigência de COVID-19. \\
\hline E3 & Estudo de coorte & $\begin{array}{l}427 \text { mulheres grávidas, submetidas à internação hospitalar entre } 1 \text { de } \\
\text { março e } 14 \text { de abril de 2020, com infecção confirmada por SARS- } \\
C o V-2 \text {. }\end{array}$ & $\begin{array}{l}\text { Descrever uma coorte nacional de gestantes internadas em hospital com infecção por } \\
\text { SARS-CoV-2 no Reino Unido, identificar fatores associados à infecção e descrever } \\
\text { resultados, incluindo a transmissão da infecção, das mães aos bebês. }\end{array}$ \\
\hline E4 & Estudo descritivo & $\begin{array}{l}\text { Gestantes/puérperas com COVID-19 e dados de etnia no banco de } \\
\text { notificação de SARS do Brasil até } 14 \text { de julho de 2020, sendo } 1860 \\
\text { brancas e } 669 \text { negras. }\end{array}$ & Descrever o impacto da COVID-19 entre mulheres gestantes negras no Brasil. \\
\hline E5 & Estudo descritivo & $\begin{array}{l}44 \text { gestantes/puérperas com COVID-19, atendidas no Brigham and } \\
\text { Women's Hospital, de } 15 \text { de março a } 1 \text { de maio de } 2020 .\end{array}$ & $\begin{array}{l}\text { Descrever sobre as disparidades raciais e étnicas na gravidade da COVID-19 em mulheres } \\
\text { grávidas nos Estados Unidos. }\end{array}$ \\
\hline E6 & Estudo descritivo & $\begin{array}{l}2475 \text { gestantes/puérperas com diagnóstico de COVID-19, notificadas } \\
\text { a partir do banco de dados oficial do Sistema de Vigilância da SARS } \\
\text { até } 14 \text { de julho de } 2020 \text {. }\end{array}$ & $\begin{array}{l}\text { Avaliar se os fatores de risco clínicos e sociais estão associados a resultados negativos para } \\
\text { a COVID-19 entre gestantes e puérperas brasileiras. }\end{array}$ \\
\hline
\end{tabular}

Fonte: Autores (2021). 
Quadro 3 - Caracterização dos artigos incluídos na revisão integrativa segundo resultados e conclusões dos artigos.

\begin{tabular}{|c|c|c|}
\hline Identificação & Resultados & Conclusões \\
\hline E1 & $\begin{array}{l}\text { 70,7\% das participantes eram de minorias raciais e grupos étnicos. A taxa de infecção por } S A R S-C o V-2 \text { na } \\
\text { gravidez foi de } 13,9 / 1.000 \text { partos (IC95\% = 8,3-23,2) em comparação com 7,3/1.000 (IC95\%=7,2-7,4) } \\
\text { em adultos de 20-39 anos. A proporção de casos de } S A R S-C o V-2 \text { na gravidez entre grupos raciais/étnicos } \\
\text { não brancos foi 2-4 vezes maior, quando comparados a outras mulheres, com pior resultado entre } \\
\text { hispânicas, índias americanas ou nativas do Alasca. }\end{array}$ & $\begin{array}{l}\text { Foram frequentes gestantes de quase todos os grupos de minorias } \\
\text { raciais/étnicas e frequentemente os cuidados médicos foram prestados usando } \\
\text { idioma diferente do inglês. As mulheres grávidas não foram protegidas do } \\
\text { COVID-19 nos primeiros meses da pandemia, com a maior carga de infecções. } \\
\text { Esses dados, apontam que a gravidez é fator de risco para doenças graves e } \\
\text { mortalidade materna, sugere que as gestantes devem ser priorizadas para vacina } \\
\text { COVID-19. }\end{array}$ \\
\hline E2 & $\begin{array}{l}\text { O grupo foi predominantemente constituído por população afro-americana. No total, } 82 \% \text { das gestantes } \\
\text { com COVID-19 eram assintomáticas. Das quatro gestantes sintomáticas, três necessitaram de oxigênio } \\
\text { suplementar, mas nenhuma necessitou de ventilação invasiva. }\end{array}$ & $\begin{array}{l}\text { COVID-19 não parece aumentar a morbidade e mortalidade entre mulheres } \\
\text { grávidas em população predominantemente afro-americana. }\end{array}$ \\
\hline E3 & $\begin{array}{l}233(56 \%) \text { das gestantes eram negras ou de outros grupos de minoria étnica; } 281 \text { (69\%) estavam com } \\
\text { sobrepeso ou eram obesas; } 175 \text { ( } 41 \%) \text { tinham } 35 \text { anos ou mais e } 145 \text { (34\%) tinham comorbidades pré- } \\
\text { existentes. Houve associação entre COVID-19 e raça, sobrepeso/obesidade e idade elevada. A chance de } \\
\text { COVID-19 entre pretas foi oito vezes maior que entre as brancas. No total, } 266 \text { (62\%) mulheres } \\
\text { evoluíram para resolução da gravidez e, destas, } 196(73 \%) \text { tiveram parto a termo; } 41 \text { mulheres (10\%) } \\
\text { necessitaram de suporte ventilatório e cinco mulheres (1\%) morreram. }\end{array}$ & $\begin{array}{l}\text { Foi alta a proporção de mulheres negras ou de grupos étnicos minoritários } \\
\text { admitidos com COVID-19 em hospital, fato que precisa ser melhor investigado } \\
\text { com urgência. }\end{array}$ \\
\hline $\mathrm{E} 4$ & $\begin{array}{l}\text { Mulheres negras internaram em piores condições, apresentando dispneia e menor saturação de } \mathrm{O}_{2} \text {, mais } \\
\text { frequentemente precisaram de UTI e de ventilação mecânica. A mortalidade materna em mulheres negras } \\
\text { devido ao COVID-19 foi quase duas vezes maior do que a observada em mulheres brancas. }\end{array}$ & $\begin{array}{l}\text { No Brasil, a intersecção de gênero, raça e classe social aprofunda a tragédia das } \\
\text { mortes maternas por COVID-19, principalmente porque o país não está } \\
\text { adotando medidas eficazes. }\end{array}$ \\
\hline E5 & $\begin{array}{l}\text { Mulheres hispânicas representaram 48\% dos casos e mulheres negras não hispânicas } 34 \% \text { dos casos, } \\
\text { valores superiores ao estimado quando se consideram problemas clínicos: } 30 \% \text { não hispânicas negras, } \\
30 \% \text { hispânicas, } 20 \% \text { não hispânicas brancas e } 15 \% \text { asiáticas. Das nove mulheres que necessitaram de } \\
\text { hospitalização para COVID-19, oito foram identificadas como negras não hispânicas ou hispânicas. Das } \\
\text { cinco pacientes com doença grave ou crítica, duas eram negras não hispânicas e duas eram hispânicas. }\end{array}$ & $\begin{array}{l}\text { Os achados do estudo refletem as consequências para a saúde dos efeitos } \\
\text { sociais, ambientais e estruturais do racismo nos Estados Unidos, incluindo } \\
\text { diferenças na prevalência de condições crônicas subjacentes e o impacto } \\
\text { desproporcional dos determinantes socioeconômicos da saúde. }\end{array}$ \\
\hline
\end{tabular}




\begin{tabular}{|c|c|c|}
\hline & $\begin{array}{l}\text { Duas gestantes necessitaram de internação em UTI e ventilação mecânica, sendo uma negra não hispânica } \\
\text { e uma hispânica. No total, foram } 25 \text { dias de internação para cinco mulheres hispânicas e } 31 \text { dias de } \\
\text { internação para as três mulheres negras não hispânicas. }\end{array}$ & \\
\hline E6 & $\begin{array}{l}\text { Análise multivariada indicou os seguintes fatores de risco independentes para resultados adversos da } \\
\text { COVID-19: estar no período pós-parto; ter idade acima de } 35 \text { anos, ter obesidade ou diabetes; ser da raça } \\
\text { negra; viver em área periurbana, sem acesso à Estratégia Saúde da Família e residir mais de 100km } \\
\text { distante do hospital de notificação do caso. Resultados adversos incluíram o óbito e a necessidade de } \\
\text { internação em UTI ou de ventilação mecânica. }\end{array}$ & $\begin{array}{l}\text { Fatores clínicos, sociais e relativos ao acesso aos serviços de saúde associaram- } \\
\text { se a resultados adversos. Entre outros, ser gestante ou puérpera de cor negra, foi } \\
\text { fator de risco independente para resultados adversos da COVID-19. }\end{array}$ \\
\hline
\end{tabular}

Fonte: Autores (2021). 


\section{Discussão}

Os dados discutidos nesse estudo voltaram-se a busca de evidências sobre a evolução da COVID-19 segundo cor da pele em gestantes/puérperas. A hipótese sob teste era que gestantes/puérperas pretas e pardas têm piores desfechos que as brancas, necessitando mais frequentemente de internação em UTI e/ou evoluindo para o óbito.

Foram identificados seis estudos, que foram realizados em três locais: EUA (E1, E2 e E5), Reino Unido (E3) e Brasil (E4 e E6), apenas um deles (E3) foi classificado com nível de evidência 4 e os demais de nível 6. Assim, considera-se pequena a produção sobre cor da pele/raça no contexto da COVID-19, com restrita distribuição no mundo e baixo nível de evidência.

Entre os estudos americanos, o número de participantes pode ser considerado pequeno: 34 binômios mãe-bebê (E2), 44 (E5) e 240 (E1) mulheres. Entre estes, diferentes grupos étnicos foram incluídos e não apenas mulheres pretas/pardas foram comparadas a brancas, como esperado. A presença de minorias raciais e diferentes grupos étnicos, como indígenas, hispânicas e nascidas em Ilhas do Pacífico, foi atestado pela necessidade de atendimento em língua não inglesa (E1) e os outros dois estudos realizados nos EUA voltaram-se a grupo afro-americano (E2) e hispânicas e/ou negras foram abordadas em conjunto (E5). A taxa de infecção de $S A R S-C o V$-2 foi significativamente maior em mulheres grávidas em comparação à população não grávida de idade semelhante e quase todos os grupos raciais/étnicos não brancos foram afetados de forma desproporcional. Considerando a elevada frequência da doença entre gestantes, quando comparadas à população geral, foi apontado que elas devem ter prioridade de vacinação (Lokken et al., 2020). O estudo constituído por gestantes/puérperas hispânicas e não hispânicas, pretas/pardas (E5) encontrou que das nove mulheres que precisaram ser hospitalizadas em decorrência da COVID19, oito eram hispânicas ou não hispânicas pretas, estas mulheres evoluíram com maior gravidade e mais frequentemente precisaram de UTI e ventilação mecânica, porém, neste caso também não houve a comparação entre pretas/pardas e brancas. Destaca-se, porém, que as mulheres pretas não hispânicas ficaram mais dias na UTI e tinham mais frequentemente comorbidades, como doença pulmonar crônica, diabetes, hipertensão arterial ou obesidade, indicando aos autores que essa situação pode ter contribuído para o aumento de sua vulnerabilidade à COVID-19. Destaca-se, também, que os autores discutiram o racismo americano, tratando em conjunto o grupo de mulheres hispânicas e não hispânicas pretas (Onwuzurike; Diouf K; Nour, 2020). Porém, em contraponto aos dois estudos que encontraram diferença de resultado segundo cor da pele/raça (E1 e E5), o estudo realizado em Detroit (E2), com população predominantemente afro-americana, concluiu que a COVID-19 não parece aumentar a morbidade e a mortalidade entre mulheres grávidas e seus recém-nascidos (Jani et al., 2020). Assim, o conjunto de estudos americanos não são conclusivos quanto ao maior risco de desfechos desfavoráveis de gestantes/puérperas pretas/pardas com COVID-19 em relação às brancas, tampouco com relação ao maior risco de gestantes e recém-nascidos a essa doença.

Estudo nacional de vigilância à saúde do Reino Unido (E3) mostrou que a maioria das gestantes internadas por COVID 19 eram pretas e de outros grupos de minorias étnicas (56\%). Houve associação entre COVID 19 e raça, sendo que a chance de ocorrência de COVID-19 foi oito vezes maior entre pretas, quando comparadas às brancas. Os autores ponderaram que os achados podem indicar maior risco de infecção e/ou de evolução para formas graves entre pretas e outros grupos minoritários, como asiáticas em geral, chinesas e mestiças, mas apontaram a necessidade de outros estudos que busquem explicar esse achado. Sugerem que o resultado não pode ser explicado simplesmente pelo maior número de casos em áreas metropolitanas que contemplam grandes proporções de mulheres de grupos étnicos minoritários, pois o pior resultado se manteve mesmo quando as áreas metropolitanas foram excluídas da análise. Para explicar a ocorrência mais frequente de COVID 19 entre gestantes pretas e outras minorias, os autores (Knight et al., 2020) citaram pesquisadores americanos, que estudando população não gestante, atribuíram o pior resultado destes grupos a diferenças nos comportamentos sociais e de saúde, presença de comorbidades, influências genéticas e de acesso aos serviços de saúde (Pareek et al., 2020). Sobre o acesso, 
ponderaram que como as disparidades foram encontradas em um país com sistema de saúde gratuito e universal, essa explicação precisa ser vista com cautela (Knight et al., 2020). Outros aspectos merecem ser mencionados: a necessidade de internação frequentemente ocorreu no final do segundo e no terceiro trimestres, indicando a necessidade de proteção durante toda a gravidez e, de modo geral, foi considerada boa a evolução materna-fetal: a maioria das mulheres não teve doença grave e foi discutido que embora a transmissão vertical possa ocorrer, é incomum (Knight et al., 2020). Assim, os resultados obtidos neste estudo inglês também não são conclusivos e os achados não foram completamente explicados.

Dois estudos brasileiros, realizados com o mesmo banco de dados de vigilância à saúde com abrangência populacional (E4, E6), encontrou proporção de gestantes/puérperas brancas e pretas/pardas de 3:1, respectivamente. Embora as médias de idade e os perfis de morbidade tenham sido semelhantes entre os dois grupos, houve pior evolução da COVID 19 entre pretas (E4): hospitalização em condição de maior gravidade; maiores taxas de admissão em UTI, de uso de ventilação mecânica e de óbito. Os pesquisadores apontaram que os resultados indicam que processos originados fora do hospital podem afetar as gestantes/puérperas pretas/pardas de maneira desproporcional, impactando na evolução da COVID-19, decorrência da intersecção entre gênero, raça e classe social (Santos et al., 2020). Em análise multivariada (E6), gestantes/puérperas de cor preta, de maneira independente, apresentaram risco 50\% maior de resultados adversos associados à COVID-19, quando comparadas às brancas (Menezes et al., 2020). Assim, em ambos os estudos brasileiros foi pior o resultado entre gestantes/puérperas pretas com COVID-19, comparadas às brancas.

Em síntese, após análise dos seis artigos incluídos nesta revisão (E1-E6), identifica-se a necessidade de outros estudos antes de se atestar o efeito independente da cor/raça preta/parda na evolução negativa da COVID-19 globalmente. Nesta revisão foram incluídos estudos observacionais, com amostras pequenas e grupos étnicos variados, não permitindo conclusões efetivas. Destaca-se, porém, que os dois estudos brasileiros que utilizaram banco de dados secundários e de base populacional, encontraram a raça/cor preta como fator de risco independentemente associado à gravidade da COVID-19.

\section{Considerações finais}

Conclui-se que, com os estudos analisados, não é possível estabelecer clara relação entre raça/cor e desfechos negativos (necessidade de UTI/óbito) da COVID-19 entre gestantes/puérperas. Devido à natureza evolutiva da pandemia, os dados estatísticos sobre gestantes e puérperas, entre elas, as mulheres pretas/pardas, são ainda limitados. Entretanto, a compreensão desse fenômeno demonstra-se necessária, pois historicamente o gênero, a raça e a classe social são componentes importantes no domínio e resolução de disparidades em saúde.

As conclusões científicas ainda são frágeis sobre COVID-19 segundo cor da pele, em verdade é preciso dar cada vez mais corpo ao debate científico, político e coletivo sobre a COVID-19 em gestantes/puérperas pretas e pardas. Portanto, é urgente nossa tarefa para compreender e agir sobre os impactos da COVID-19 na vida das mulheres gestantes/puérperas pretas, para a elaboração de protolocos, diretrizes e políticas que deem conta de garantir todas as vidas de gestantes pretas. Assim, outras investigações são necessárias, para que se possa compreender como a questão central desse estudo, a evolução da COVID-19 segundo cor/raça, se apresenta globalmente.

\section{Referências}

Alfaraj, S. H., AL-Tawfiq, J. A. \& Memish, Z. A. (2019). Middle East Respiratory Syndrome Coronavirus (MERS-CoV) infection during pregnancy: Report of two cases \& review of the literature. Journal of Microbiology, Immunology, and Infection. 52(3), 501-3. https://dx.doi.org/10.1016\%2Fj.jmii.2018.04.005

Amorim, M. M. R., Takemoto, M. L. S. \& Fonseca, E. B. (2020). Maternal deaths with coronavirus disease 2019: a different outcome from low- tomiddleresource countries? Am J Obstet Gynecol. https://doi.org/10.1016/j.ajog.2020.04.023

Brasil, Ministério da Saúde. (2020a). Resposta nacional e internacional de enfrentamento ao novo coronavírus. https://coronavirus.saude.gov.br/linha-dotempo/\#dez2019 
Brasil, Ministério da Saúde. Secretaria de Vigilância em Saúde. (2020b). Doença pelo Coronavírus COVID-19. Boletim Epidemiológico Especial - Semana Epidemiológica (SE) 33 (08 a 15/08). https://saude.gov.br/images/pdf/2020/August/19/Boletim-epidemiologico-COVID-27.pdf

Brasil, Ministério da Saúde. Secretaria de Atenção Primária à Saúde. Departamento de Ações Programáticas e Estratégicas. (2020c). Nota Informativa nº 13/2020 - SE/GAB/SE/MS - Manual de Recomendações para a Assistência à Gestante e Puérpera frente à Pandemia de Covid-19 [recurso eletrônico] / Ministério da Saúde, Secretaria de Atenção Primária à Saúde. - Brasília: Ministério da Saúde. http://189.28.128.100/dab/docs/portaldab/documentos/corona/manual_recomendacoes_gestantes_covid19.pdf

Breslin, N., Baptiste, C., Miller, R., Fuchs, K., Goffman, D., Gyamfi-Bannerman, C. \& D'Alton, M. (2020). COVID-19 in pregnancy: early lessons, American Journal of Obstetrics \& Gynecology MFM. https://doi.org/10.1016/j.ajogmf.2020.100111

Chen, H., Guo, J., Wang, C., Luo, F., Yu, X., Zhang W et al. (2020). Clinical characteristics and intrauterine vertical transmission potential of COVID-19 infection in nine pregnant women: a retrospective review of medical records. Lancet. https://doi.org/10.1016/S0140-6736(20)30360-3

Collin, J., Bystrom, E., Carnahan, A. \& Ahrne , M. (2020). Public Health Agency of Sweden's brief report: pregnant and postpartum women with SARS-CoV2 infection in intensive care in Sweden. Acta Obstet Gynecol Scand. https://doi.org/10.1111/aogs.13901

Ellington, S., Strid, P., Tong, V.T et al. (2020). Characteristics of Women of Reproductive Age with Laboratory-Confirmed SARS-CoV-2 InfectionbyPregnancy Status - United States, January 22-June 7, MMWR Morb Mortal Wkly Rep. 2020; 69: 769-75. http://dx.doi.org/10.15585/mmwr.mm6925a1external\%20icon

Ercole, F.F., Melo L.S. \& Alcoforado, C.L.G.C. (2014). Revisão integrativa versus sistemática. Rer Min Enferm. 2014;18(1):10. https://doi.org/10.5935/14152762.20140001

Galvão, C.M., Sawada, N.O. \& Trevizan, M.T. (2004). Revisão sistemática: recurso que proporciona a incorporação das evidências na prática da enfermagem. Revista Latino-Americana de Enfermagem, 12(3), 549-556. https://doi.org/10.1590/S0104-11692004000300014

Ganong, L.H. (1987). Revisões integrativas da pesquisa em enfermagem. Res Nurs Health. 1987; 10 (1): 1-11. https://doi.org/10.1002/nur.4770100103

Goes, E.F., Ramos, D.O. \& Ferreira, A.J.F. (2020). Desigualdades raciais em saúde e a pandemia da Covid-19. Trabalho, Educação e Saúde, 18(3), e00278110. Epub May 29. https://doi.org/10.1590/1981-7746-sol00278

Jani, S., Jacques, S.M., Qureshi, F., Natarajan, G., Bajaj, S., Velumula, P., Agu, C. \& Bajaj M. (2021). Clinical Characteristics of Mother-Infant Dyad and Placental Pathology in COVID-19 Cases in Predominantly African American Population. AJP Rep. Jan;11(1):e15-e20. http://dx.doi.org/10.1055/s-00401721673

Knight, M., Bunch, K., Vousden, N., Morris, E., Simpson, N., Gale, C et al. (2020). Characteristics and outcomes of pregnant women admitted to hospital with confirmed SARS-CoV-2 infection in UK: national population based cohort study. BMJ; 369: m2107. https://doi.org/10.1136/bmj.m2107

Li, N. et al. (2020). Maternal and neonatal outcomes of pregnant women with COVID-19 pneumonia: a case-control study. Clinical Infectious Diseases, [s. 1.]. https://doi.org/10.1093/cid/ciaa352

Liu, D., Li, L., Wu, X., et al. (2020a). Pregnancy and perinatal outcomes of women with COVID-19 Pneumonia: a preliminary analysis. AJR Am J Roentgenol. https://doi.org/10.2214/AJR.20.23072

Liu, Y., Chen, H., Tang, K. \& Guo Y. (2020b). Clinical manifestations and outcome of SARS-CoV-2 infection during pregnancy [published online ahead of print, 2020b Mar 4]. J Infect. https://doi.org/10.1016/j.jinf.2020.02.028

Lokken, E.M et al. (2021). Higher SARS-CoV-2 Infection Rate in Pregnant Patients. American journal of obstetrics and gynecology, S0002-9378(21)00098-3. 11 Feb. 2021. http://dx.doi.org/10.1016/j.ajog.2021.02.011

Lumbreras-Marquez, M.I., Campos-Zamora, M., Lizaola-Diaz de Leon, H. \& Farber, M.K. (2020). Maternal mortality from COVID-19 in Mexico. Int J Gynaecol Obstet. 2020; 150: 266-7. https://doi.org/10.1002/ijgo.13250

Melnyk, B.M., Fineout-Overholt, E. (2005). Making the case for evidence-based practice.In: Melnyk BM, Fineout-Overholt E. Evidence based practice in nursing \& healthcare. A guide to best practice. Philadelphia: Lippincot Williams \& Wilkins; p.3-24

Menezes, M.O., Takemoto, M.L.S., Nakamura-Pereira, M., Katz, L., Amorim, M.M.R., Salgado, H.O., Melo, A., Diniz, C.S.G., de Sousa, L.A.R., Magalhaes, C.G., Knobel, R. \& Andreucci, C.B; (2020). Brazilian Group of Studies for COVID-19, Pregnancy. Risk factors for adverse outcomes among pregnant and postpartum women with acute respiratory distress syndrome due to COVID-19 in Brazil. Int J Gynaecol Obstet. Dec;151(3):415-423. http://dx.doi.org/10.1002/ijgo.13407

Nakamura-Pereira, M., Amorim, M.M.R., Pacagnella, R.C., Takemoto, M.L.S., Penso, F.C.C., Rezende-Filho, J et al. (2020). COVID-19 and Maternal Death in Brazil: An Invisible Tragedy. RevBrasGinecol Obstet. 2020;42(8):445-7. https://doi.org/10.1055/s-0040-1715138

Organização das Nações Unidas das Mulheres do Brasil. (2020). Mulheres Negras agem para enfrentar o racismo na pandemia Covid-19 e garantir direitos da população negra no "novo normal". http://www.onumulheres.org.br/noticias/mulheres-negras-agem-para-enfrentar-o-racismo-na-pandemia-covid-19-egarantir-direitos-da-populacao-negra-no-novo-normal/

Organização das Nações Unidas. (2020). Racismo aumenta exposição de pessoas negras à COVID-19 e limita atendimento, afirmam pesquisadoras. https://nacoesunidas.org/racismo-aumenta-exposicao-de-pessoas-negras-a-covid-19-e-limita-atendimento-afirmam-pesquisadoras/

Onwuzurike, C., Diouf K, M.A.R. \& Nour, N.M. (2020). Racial and ethnic disparities in severity of COVID-19 disease in pregnancy in the United States. Int J Gynaecol Obstet. Nov;151(2):293-295. http://dx.doi.org/10.1002/ijgo.13333 
Research, Society and Development, v. 10, n. 6, e6710615531, 2021 (CC BY 4.0) | ISSN 2525-3409 | DOI: http://dx.doi.org/10.33448/rsd-v10i6.15531

Pareek, M., Bangash, M.N., Pareek, N., Pan, D., Sze, S., Minhas, J.S et al. (2020). Ethnicity and covid-19: an urgent public health research priority. Lancet. 395:1421-2. https://doi.org/10.1016/S0140-6736(20)30922-3

Santos, M.P.A et al. (2020). População negra e Covid-19: reflexões sobre racismo e saúde. Estud. av., São Paulo, v. 34, n. 99, p. 225-244, Aug. https://doi.org/10.1590/s0103-4014.2020.3499.014

Souza, M.T., Silva, MD. \& Carvalho, R. (2010). Revisão integrativa: o que é e como fazer. Einstein (São Paulo), 8(1), 102-106. https://doi.org/10.1590/s167945082010rw1134

Takemoto, M.L.S., Menezes, M.D.O., Andreucci, C.B., Nakamura-Pereira, M., Amorim, M.M., Katz, L. \& Knobel, R. (2020). The tragedy of COVID-19 in Brazil: 124 maternal deaths and counting. Int J Gynecol Obstet. https://doi.org/10.1002/ijgo.13300

Vivanti, A.J., Mattern, J., Vauloup-Fellous, C., Jani, J., Rigonnot, L., El Hachem, L et al. (2020). Retrospective Description of Pregnant Women Infected with Severe Acute Respiratory Syndrome Coronavirus 2, France. EmergInfectDis. 26 (9): 2069-76. https://wwwnc.cdc.gov/eid/article/26/9/20-2144_article

Zaigham, M., Andersson, O. (2020). Maternal and Perinatal Outcomes with Covid-19: a systematic review of 108 pregnancies. Acta Obstetricia Et Gynecologica Scandinavica, [s. 1.]. https://doi.org/10.1111/aogs.13867

Zambrano, L.D., Ellington, S., Strid, P., Galang, R.R., Oduyebo, T., Tong., V.T, Woodworth, K.R., Nahabedian, J.F., Azziz-Baumgartner, E., Gilboa, S.M. \& Meaney-Delman, D. (2020). Update: Characteristics of Symptomatic Women of Reproductive Age with Laboratory-Confirmed SARS-CoV-2 Infection by Pregnancy Status - United States, January 22-October 3, 2020. Weekly / November 6, 2020. MMWR Morb Mortal Wkly Rep. 9(44);1641-1647. https://doi.org/10.15585/mmwr.mm6944e3

Yu, N., Li, W., Kang, Q., Xiong, Z., Wang, S., Lin, X et al. (2020). Clinical features and obstetric and neonatal outcomes of pregnant patients withCOVID-19 in Wuhan, China: a retrospective, single-centre, descriptive study. Lancet Infect Dis. https://doi.org/10.1016/S1473-3099(20)30176-6

Wang, X., Zhou, Z., Zhang, J., Zhu, F., Tang, Y. \& Shen, X. (2020). A case of 2019 Novel Coronavirus in a pregnant woman with preterm delivery [published online ahead of print, 2020 Feb 28]. Clin Infect Dis. https://doi.org/10.1093/cid/ciaa200

Wong, S. F., Chow, K. M., Leung, T. N., Ng, W. F., Ng, T. K., Shek, C. C. \& Tan, P. Y. (2004). Pregnancy and perinatal outcomes of women with severe acute respiratory syndrome. American Journal of Obstetrics and Gynecology, 191(1), 292-297. https://doi.org/10.1016/j.ajog.2003.11.019

World Health Organization. (2020). Coronavirus disease 2019 (Covid19). Situation Report (SR) - 51, 2020. https://www.who.int/docs/defaultsource/coronaviruse/situation-reports/20200311-sitrep-51-covid-19.pdf?sfvrsn=1ba62e57_10

World Health Organization. (2020). Coronavirus disease 2019 (Covid19). Situation Report (SR) - 81, 2020. https://www.who.int/docs/defaultsource/coronaviruse/situation-reports/20200410-sitrep-81-covid 19.pdf?sfvrsn=ca96eb84_2 